\title{
Age-related decline of myelin proteins is highly correlated with activation of astrocytes and microglia in the rat CNS
}

\author{
FANG XIE ${ }^{1,2^{*}}$, JIU-CONG ZHANG ${ }^{3 *}, \mathrm{HAN} \mathrm{FU}^{1 *}$ and JUN CHEN ${ }^{1}$ \\ ${ }^{1}$ Institute for Biomedical Sciences of Pain, Tangdu Hospital, The Fourth Military Medical University, \\ Xi'an, Shaanxi 710038; ${ }^{2}$ Department of Stress Medicine, Institute of Basic Medical Sciences, \\ Academy of Military Medicine Sciences, Beijing 100850; ${ }^{3}$ Department of Gastroenterology, \\ Lanzhou General Hospital of Lanzhou Military Command, Lanzhou, Gansu 730050, P.R. China
}

Received May 18, 2013; Accepted August 19, 2013

DOI: $10.3892 /$ ijmm.2013.1486

\begin{abstract}
It has been shown that aging can greatly influence the integrity and ultrastructure of white matter and the myelin sheath; however, studies regarding the effects of aging on the expression of myelin proteins are still limited. In the present study, immunohistochemical mapping was used to investigate the overall expression of myelin basic protein (Mbp) and myelin oligodendrocyte glycoprotein (Mog) in the central nervous system (CNS) of rats in postnatal months 2, 5, 18 and 26. Astrocyte and microglia activation was also detected by glial fibrillary acidic protein (GFAP) or ionized calciumbinding adaptor molecule 1 (Ibal) staining and western blotting. A significant decline of Mbp and Mog was identified as a universal alteration in the CNS of aged rats. Aging also induced significant astrocyte and microglial activation. Correlation analysis indicated a negative correlation between the reduction of age-related myelin proteins and glial activation in aging. This correlation of myelin breakdown and glial activation in aging may reveal new evidence in connecting the inflammation and myelin breakdown mechanism of age-related neurodegenerative diseases.
\end{abstract}

\section{Introduction}

White matter of the central nervous system (CNS) is named after its glistening pale appearance visible on the cut surface of the brain and spinal cord. This feature is attributed to the myelin sheath, which is a multiple laminar membrane structure wrapped around the axons and plays an important role,

Correspondence to: Dr Fang Xie, Tangdu Hospital, The Fourth Military Medical University, 1st Xinsi Road, Baqiao District, Xi'an, Shaanxi 710038, P.R. China

E-mail:vancoxie@sina.com

${ }^{*}$ Contributed equally

Key words: aging, myelin proteins, astrocyte, microglia, central nervous system, mapping as an insulate cable-coat, in maintaining the fast saltatory conduction of action potentials along the nerve fibers (1). The lipid-rich feature of the myelin sheath makes it very fragile to the environmental changes in the brain, such as metabolic disorders and aging. Due to the sensitivity of myelin sheath to senescence, it is thought to be one of the major targets of brain aging (2). Age-related alterations in the myelin sheath were identified in the light microscopy study of Lintl and Braak (3), who observed a reduction in the intensity of hematoxylin staining in the aged myelin. Since then, increasing evidence has suggested the existence of a progressive myelin breakdown in the aged nervous system. Many neuroimaging studies, especially the diffusion tensor imaging and the voxel-based morphometric analysis, showed an obvious age-related decline in human brain white matter integrity, in other words a reduction in the structural integrity of the myelin sheath $(4,5)$. Ultrastructure electron microscopy studies demonstrated that age-related myelin breakdown could be identified in both the central and peripheral nervous system of humans, non-human primates, rodents and other species $(6,7)$. Furthermore, the alterations of the myelin sheath in an aging brain are considered to be involved in the development of age-related disorders, such as Alzheimer's and Parkinson's disease $(8,9)$. However, the mechanism of these age-related alterations in the myelin sheath has not been fully understood.

Myelin-associated proteins, such as myelin basic protein (Mbp) and proteolopid protein (Plp), are key components of the myelin sheath and play a very important role in maintaining the integrity of myelin multi-lamellar structure. However, investigations concerning the expression level of myelin proteins in aged CNS are limited, for both humans and animals. On the other hand, there is general agreement that astrocyte and microglia undergo activation with age in rodents, monkeys and even humans. These two types of glial cells become hypertrophic in an aged brain, express more senescence markers and occasionally increase their numbers $(10,11)$. Nevertheless, it is not fully understood whether this activation of astrocytes and microglia contributes to the age-related myelin breakdown. In the present study, we investigated the age-related expression of myelin proteins using a whole CNS mapping. The downregulation of Mbp and myelin oligodendrocyte glycoprotein (Mog) was observed as 
a general alteration of the myelin sheath in aging. Age-related activation of astrocytes and microglia was also analyzed. The negative correlation between the myelin protein breakdown and glial cell activation was identified.

\section{Materials and methods}

Animals. Male Sprague-Dawley albino rats ranging in age from postnatal month (PNM) 2-26 were used. The rats were divided into 4 groups of 7 animals each, aged PNM2, 5, 18 and 26. All the animals were provided by the Laboratory Animal Center of the Fourth Military Medical University (FMMU). Animals were housed in plastic boxes at $22-26^{\circ} \mathrm{C}$ on a $12-\mathrm{h}$ light/dark cycle. Food and water were provided ad libitum. Drinking water was chlorinated and acidified to $\mathrm{pH}$ 6.2-6.8. Cages were cleaned with autoclaving and their environment was maintained strictly steady. The experimental protocols were approved by the Institutional Animal Care and Use Committee of FMMU (permit no.: SCXK2007-007). The present study was performed in accordance with the National Institute of Health Guide for the Care and Use of Laboratory Animals (NIH Publications no. 80-23) revised in 1996.

Immunohistochemistry. Three rats from each group were anesthetized with pentobarbital sodium (100 mg/kg, i.p.; Sigma, St. Louis, MO, USA) and transcardially perfused with $0.9 \%$ saline, followed by $4 \%$ paraformaldehyde in $0.1 \mathrm{M}$ phosphate-buffered saline (PBS). Whole brain and spinal cord were dissected, post-fixed at $4^{\circ} \mathrm{C}$ for an additional $90 \mathrm{~min}$ in the same fixative solution and transferred into $30 \%$ sucrose $0.01 \mathrm{M}$ PBS overnight. Serial $40 \mu \mathrm{m}$ cryo-sections (coronal brain sections and transversal spinal sections) were cut on CM1900 freezing microtome (Leica, Mannheim, Germany), incubated for $1 \mathrm{~h}$ with $0.05 \%$ Triton $\mathrm{X}-100$ and then with $10 \%$ goat serum in $0.01 \mathrm{M}$ PBS at room temperature, followed by incubation with the primary antibody at $4{ }^{\circ} \mathrm{C}$ overnight. The primary antibodies were mouse anti-myelin oligodendrocyte glycoprotein (anti-Mog) (1:200, Millipore, Billerica, MA, USA), rabbit anti-Mbp (1:200, Sigma), rabbit anti-glial fibrillary acidic protein (anti-GFAP) (GFAP, 1:400, Millipore) and rabbit anti-ionized calcium-binding adaptor molecule 1 (anti-Iba1) (Iba1, 1:300, Wako, Osaka, Japan). Diamidino-phenyl-indole (DAPI, $1 \mu \mathrm{g} / \mathrm{ml}$, Sigma) was administered for at least $30 \mathrm{sec}$ to stain the cell nucleus for fluorescence labeling. Micrographic images were obtained under the microscope (Olympus, Tokyo, Japan). GFAP or Iba1-positive cells were counted by Image-pro Plus software in the rat cerebral cortex S1, dentate gyrus (DG) of hippocampal formation, corpus callosum, internal capsule, spinal cord dorsal horn and spinal cord posterior funiculus, respectively. The number of DAPI-labeled cell nuclei in the corpus callosum and posterior funiculus were also obtained through Image-pro Plus software. Cell densities were calculated in the prementioned regions.

Western blotting. Four rats from each group were anesthetized with pentobarbital sodium (100 mg/kg, i.p.). The whole brain, without the cerebellum, was dissected, homogenized and lysed in an ice-cold RIPA lysis buffer (Applygen Technologies Inc., Beijing, China) of $1 \%$ NP-40, $0.1 \%$ sodium dodecyl sulphate (SDS) in $50 \mathrm{mM}$ Tris- $\mathrm{HCl}, \mathrm{pH} 7.4$ and containing protease inhibitor. Protein concentrations were determined by the BCA protein assay kit (Thermo Scientific, Pittsburgh, PA, USA). After $10 \mathrm{~min}$ of incubation at $98^{\circ} \mathrm{C}$ with SDS-PAGE buffer, protein samples $(40 \mu \mathrm{g})$ were separated by $10 \%$ SDS-PAGE gel and transferred to a polyvinylidene difluoride membrane with a semi-dry transfer system (Bio-Rad, Hercules, CA, USA). Membranes were blocked at room temperature for $1 \mathrm{~h}$ with $5 \%$ milk in PBS containing 0.2\% Tween-20, followed by incubation with antibody. Mouse anti-Mog (1:500, Millipore), rabbit anti-Mbp (1:500, Sigma), rabbit anti-GFAP (1:800, Millipore) and rabbit anti-Iba1 (1:600, Wako) antibodies served as the primary antibodies. Mouse anti-rat $\beta$-tubulin antibody (1:8,000, Sigma) was used as an internal control. The membranes were developed with Pierce ECL Western blotting substrate kit (Thermo Scientific) and the signals were captured with FluorChem ${ }^{\circledR}$ FC2 (Alpha Innotech, San Leandro, CA, USA). Scanned images were analyzed by Quantity One 1-D analysis software (Bio-Rad).

Statistical analysis. Data are expressed as means \pm SEM. Differences among groups were compared using one-way ANOVA, followed by Bonferroni's or Fisher's PLSD post-hoc analysis when appropriate. Correlation between the expression levels of GFAP, Iba1, Mbp and Mog based on western blot analysis results was analyzed by Pearson's coefficients and multiple comparisons were corrected by using Bonferroni's analysis. Multiple regression analyses, with stepwise procedure, were performed to investigate the determinants of the myelin protein decline in aged rat brains. All analyses were performed using the SPSS statistical package. Statistical significance was indicated by $\mathrm{P}<0.05$.

\section{Results}

Age-related down-regulation of myelin proteins. Using whole CNS immunohistochemical mapping, we observed a marked downregulation in the expression of myelin associated proteins, such as Mog and Mbp (Fig. 1 and data not shown). This alteration of myelin protein expression is universal in the CNS, it appears from the prefrontal section of the brain to the medulla and can be identified in any segment of the spinal cord (Fig. 1). This myelin-protein downregulation does not occur only in very old rat CNS. In fact, this alteration was first identified in PNM5 in our study (data not shown). As Mog and Mbp are usaully highly expressed in the white matter, the reduction of Mog and Mbp levels was much more obvious in the white matters of the corpus callosum in the brain and the posterior funiculus in the spinal cord (Fig. 2A-H). Immunohistochemical data showed that Mog and Mbp were highly expressed and distributed parallel to the nerve fibers in PNM2 corpus callosum. However, the expression of Mog markedly decreased and had a twisted and corrugated appearance in PNM18 corpus callosum (Figs. 2A and B, and 3A and B), while Mbp was hardly detectable (Fig. 2C and D). The posterior funiculus of the spinal cord in PNM18 lost most response to Mog and Mbp antibodies and only some Mog- and Mbp-positive cells were present (Figs. 2E-H, and 3D and E). The changes of myelin protein expression were a little complicated in the grey matters. 
A

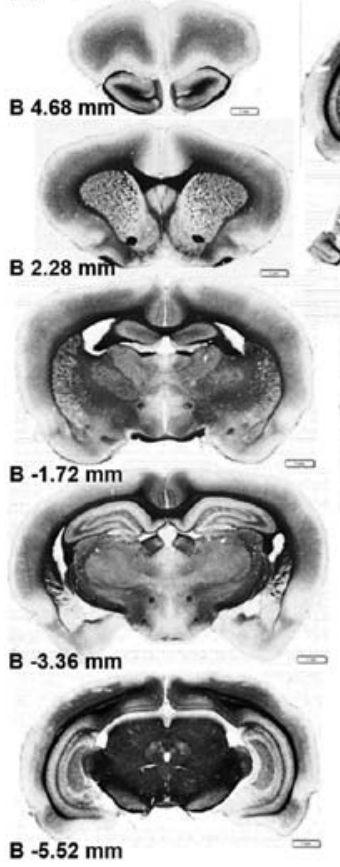

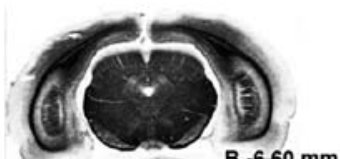
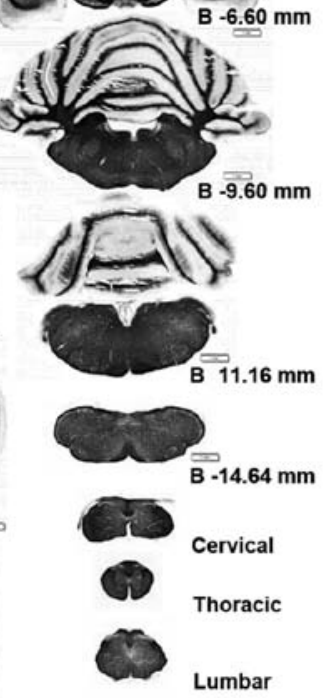

Scale bar=1 $\mathrm{mm}$
B

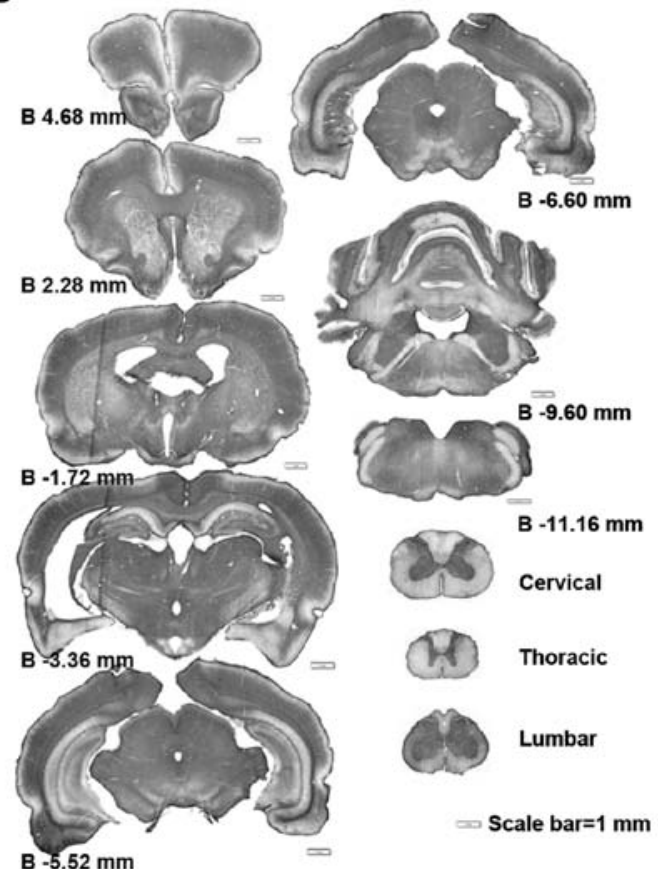

Figure 1. Myelin oligodendrocyte glycoprotein (Mog) expression altered with age in rat brain and spinal cord. Mog expression in (A) PNM2 and (B) PNM18 rat brain and spinal cord. Scale bar, $1 \mathrm{~mm}$.

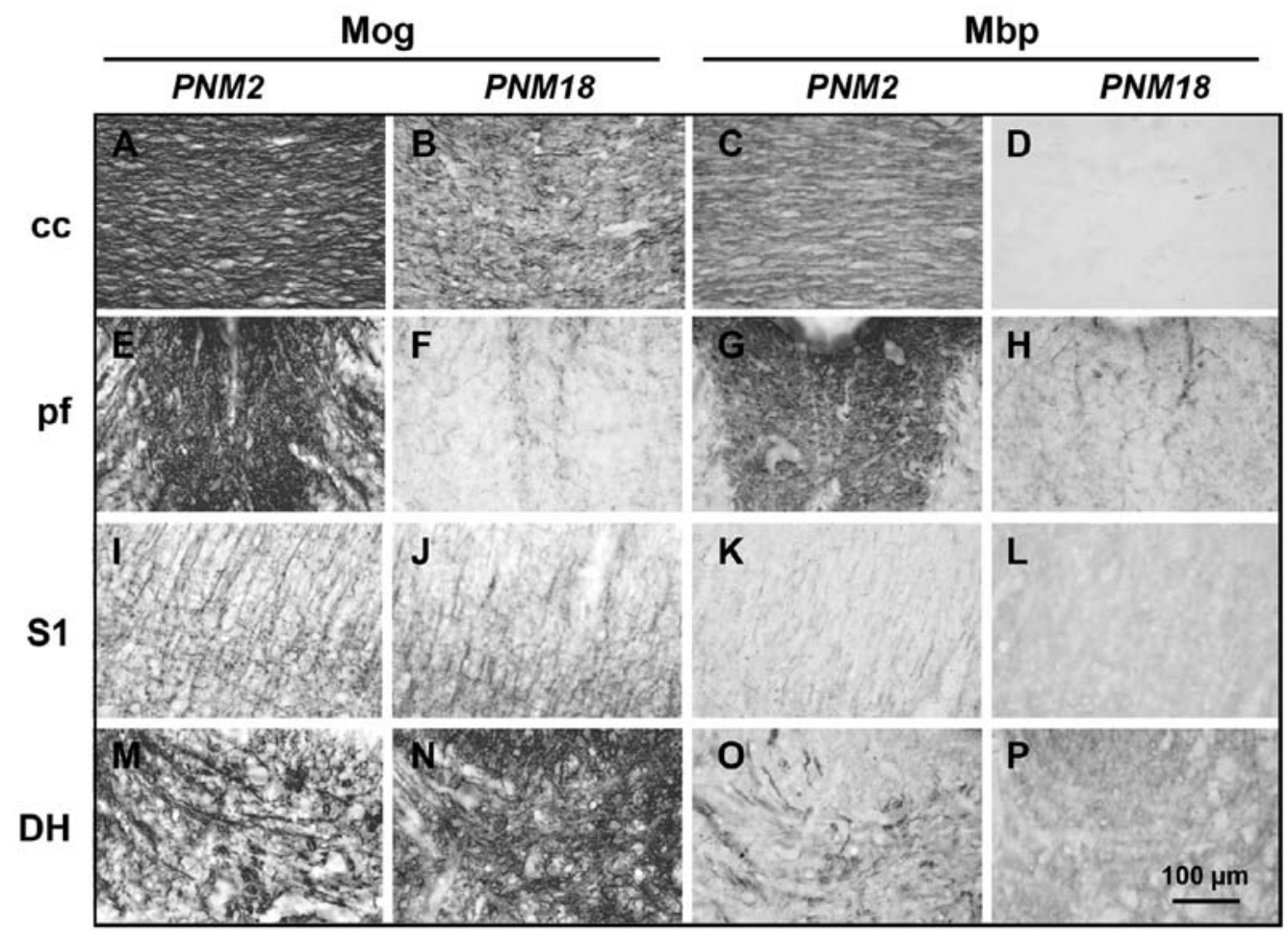

Figure 2. Age-related alteration in myelin protein expression. (A-D) Decreased expression of myelin oligodendrocyte glycoprotein (Mog) and myelin basic protein (Mbp) in aged corpus callosum (cc). (E-H) Downregulation of Mog and Mbp in spinal cord posterior funiculus (pf). (I-L) Expression of Mog and Mbp in postnatal month 2 (PNM2) and PNM18 primary somatosensory cortex (S1). (M-P) Alteration of Mog and Mbp expression in aged spinal cord dorsal horn (DH). Scale bar, $100 \mu \mathrm{m}$.

Compared with the PNM2 rat CNS, the amount of Mbp seems to be at the same level in the primary somatosensory cortex (Fig. 2K and L) and spinal cord dorsal horn (Fig. 2O and P) of PNM18 rat. Specifically, Mog was expressed even more in the aged somatosensory cortex (Fig. 2I and J) and dorsal horn (Fig. 2M and N). DAPI labeling showed that the number of glial cells, including oligodendrocytes, astrocytes and microglia, increased significantly in the aged corpus callosum. 

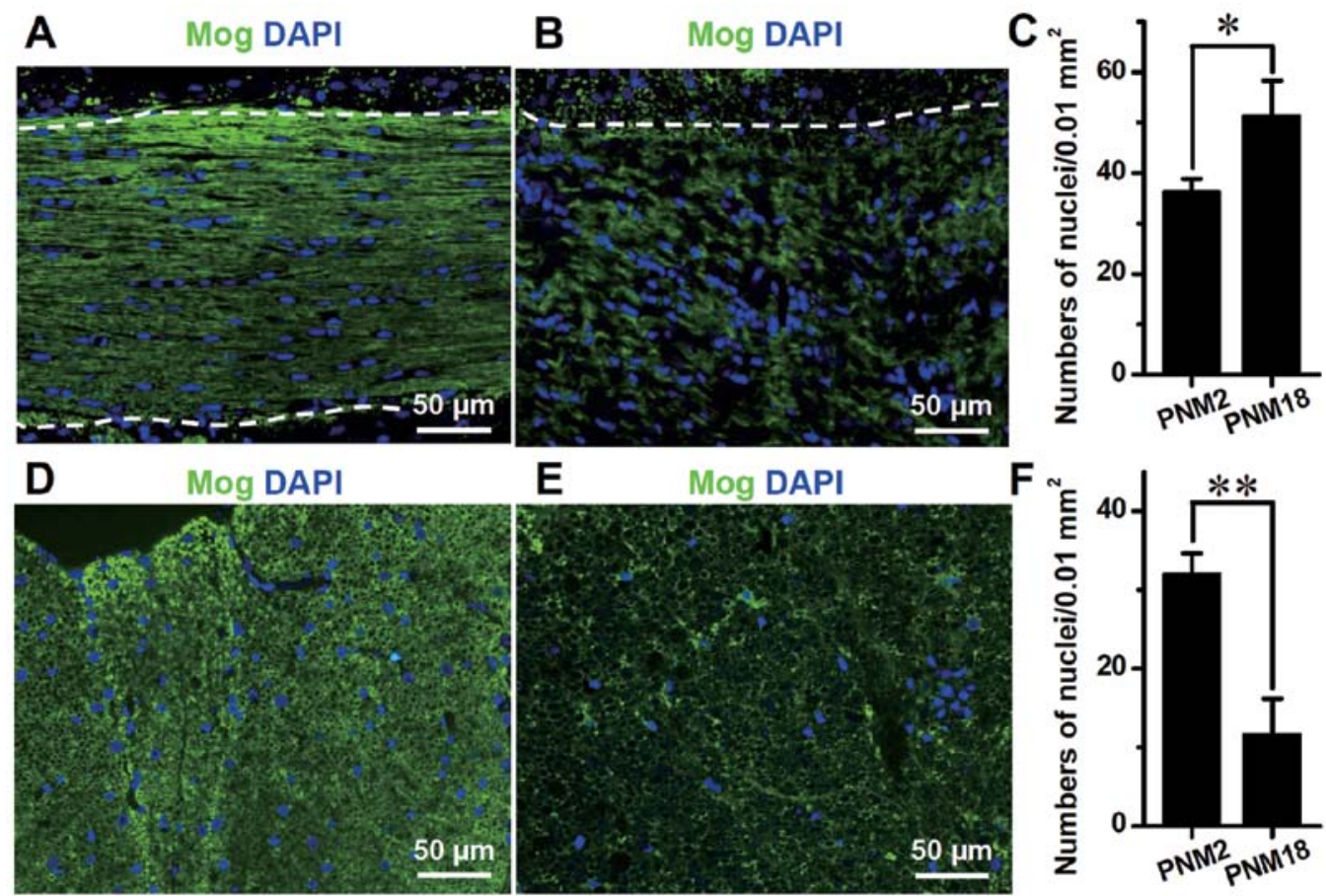

Figure 3. Myelin oligodendrocyte glycoprotein (Mog) altered with age in the rat corpus callosum and dorsal column. Expression of Mog in (A) postnatal month 2 (PNM2) and (B) PNM18 rat corpus callosum. The density of DAPI-labeled nucleus in the corpus callosum was evident in (C-E), showing the level of Mog in the PNM2 and PNM18 rat dorsal column, respectively. The density of DAPI-labeled nucleus in the spinal cord dorsal column is shown in F. Scale bar, $50 \mu \mathrm{m} ;{ }^{*} \mathrm{P}<0.05 ;{ }^{* *} \mathrm{P}<0.01$; Error bars: $\pm \mathrm{SEM}$.

A

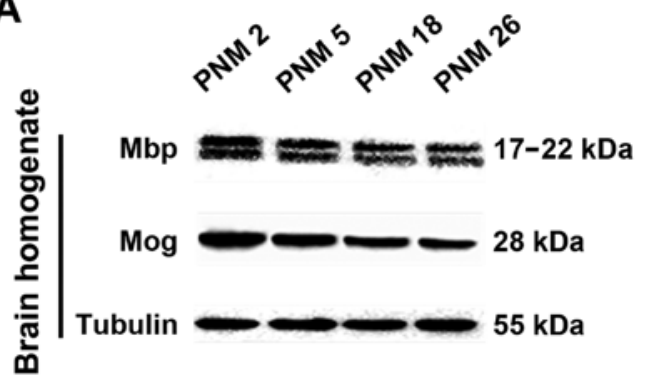

B

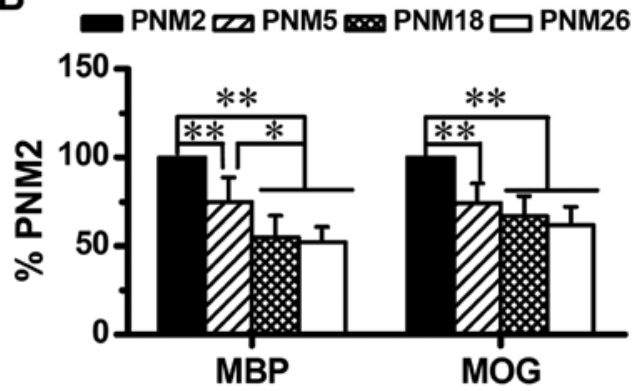

Figure 4. The downregulation of myelin protein in aging brain. (A) Expression of myelin basic protein (Mbp) and myelin oligodendrocyte glycoprotein (Mog) based on western blot analysis in postnatal month 2 (PNM2), 5, 18 and 26 rat brain tissue homogenate ( $\mathrm{n}=4$ per time point). (B) Statistical analysis of western blot results. ${ }^{*} \mathrm{P}<0.05 ;{ }^{* *} \mathrm{P}<0.01$; Error bars: \pm SEM.

Contrary to the arrangement of glial nuclei in rows between nerve fibers in PNM2, the glial nuclei in PNM18 corpus callosum were distributed more irregularly and some of them were grouped together as in a nest (Fig. 3B). By contrast, the glial nuclei decreased significantly in the posterior funiculus of aged spinal cords (Fig. 3E and F). However, linked nuclei could still be observed (Fig. 3E).

Similar to the results of immunohistochemical mapping and immunofluorescence, results of western blotting also showed an age-related alteration in the expression of Mog and Mbp in the rat brain (Fig. 4). The downregulation of Mog and Mbp is age-dependent. Compared with PNM2, the expression level of Mog and Mbp in PNM26 decreased almost 50\% (Fig. 4B).

Activation of astrocytes and microglia in aged rat CNS. The immunohistochemical mapping of the rat CNS was conducted to investigate the activation of astrocytes and microglia. GFAP and Ibal were used as activation markers of astrocytes and microglia, respectively. The mapping results showed a significant age-related increase in the expression of GFAP and Iba1 (Figs. 5 and 6). In PNM2 rat, the pale appearance of tissue slides indicated the weakness in CNS response to anti-GFAP or Iba1 antibody. The upregulation of GFAP and Iba1 was first detected in PNM5 and was also observed in PNM18 and PNM26 rat CNS. The number of GFAP-positive cells markedly increased in the grey and white matters of PNM18 rat (Fig. 5A-L and N). In the primary somatosensory cortex and dentate gyrus of hippocampal formation of PNM18, a 10-fold increase in the density of astrocyte was identified (Fig. 5A-D and M). Similarly, the GFAP labeling indicated that the density of astrocyte increased three to seven times in aged corpus callosum, internal capsule and spinal cord posterior funiculus (Fig. 5E-H and K-M). Of note, although the number of GFAP-positive cells increased in the white matters of PNM18 


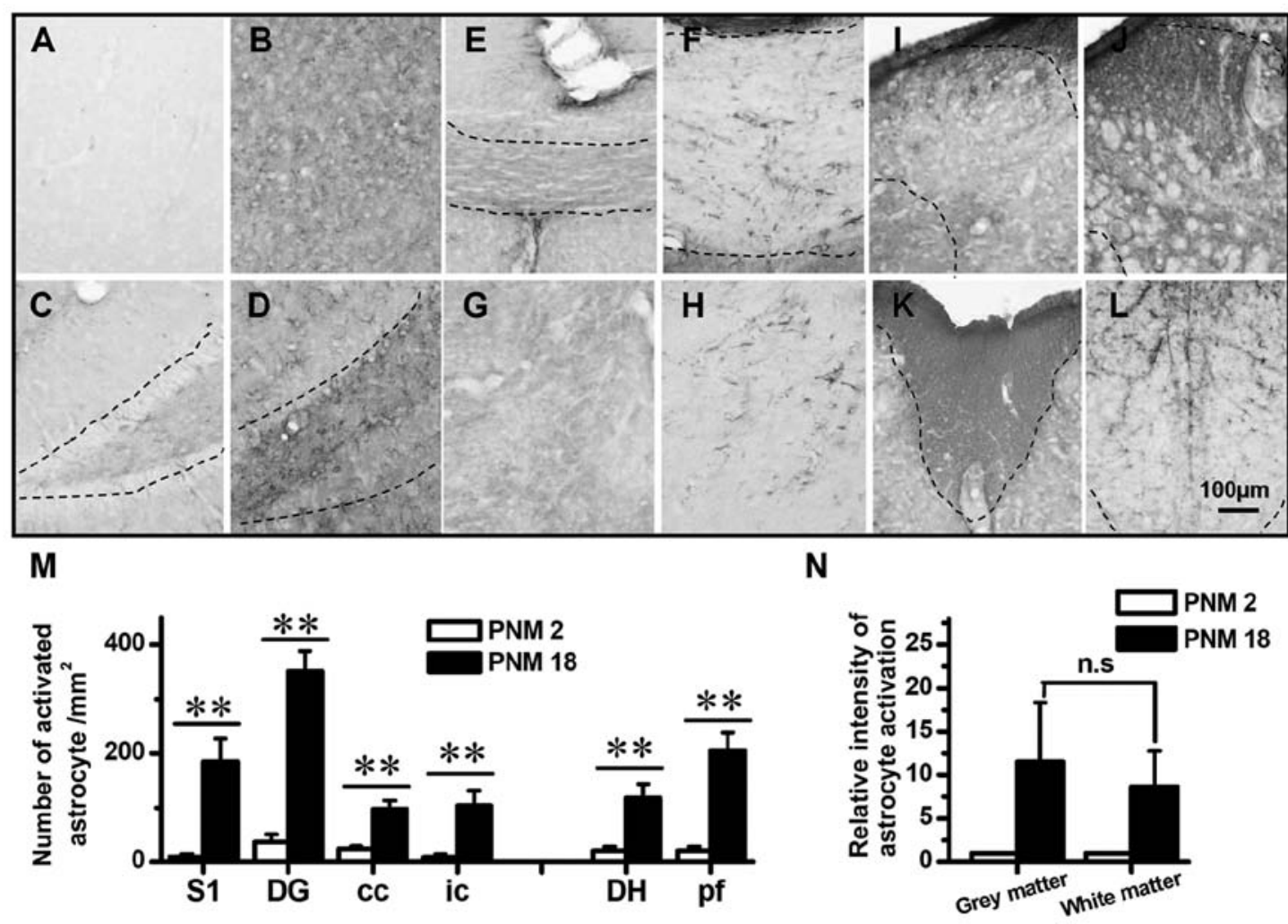

Figure 5. Astrocyte activated in aged rat brain and spinal cord. (A), (C), (E), (G), (I) and (K) show the GFAP immunochemistry staining in PNM2 rat cerebral cortex S1, dentate gyrus of hippocampal formation, corpus callosum, internal capsule, spinal cord dorsal horn and spinal cord posterior funiculus, respectively. (B), (D), (F), (H), (J) and (L) show the astrocyte in postnatal month 18 (PNM18) rat brain and spinal cord positions mentioned above. The number of activated astrocyte per square millimeter in young and aged rat brains and spinal cords are shown in $(\mathrm{M})$. (N) shows the relative intensity of astrocyte activation in aged rats (normalized by comparing with PNM2 number of activated astrocyte per square millimeter) within the grey and white matters, respectively. Scale bar, $100 \mu \mathrm{m} ;{ }^{*} \mathrm{P}<0.05 ;{ }^{* *} \mathrm{P}<0.01$ compared with PNM2; Error bars: \pm SEM.

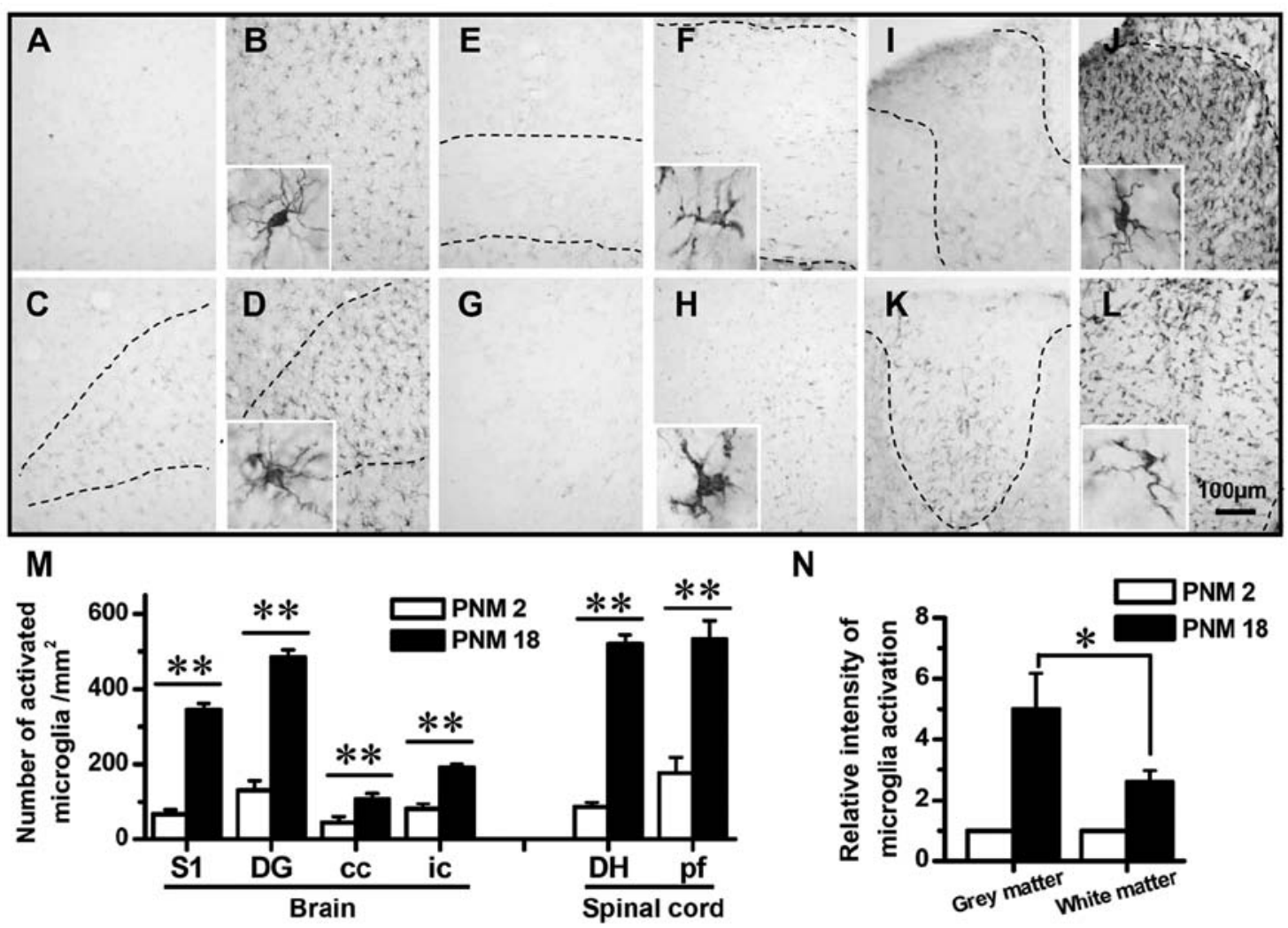

Figure 6. Microglia activated in aged rat brain and spinal cord. Ionized calcium-binding adaptor molecule 1 (Iba1) was used to visualize microglias. For region annotation see Fig. 5. Scale bar, $100 \mu \mathrm{m} ;{ }^{*} \mathrm{P}<0.05 ;{ }^{* *} \mathrm{P}<0.01$ compared with postnatal month 2 (PNM2); Error bars: \pm SEM. 
A
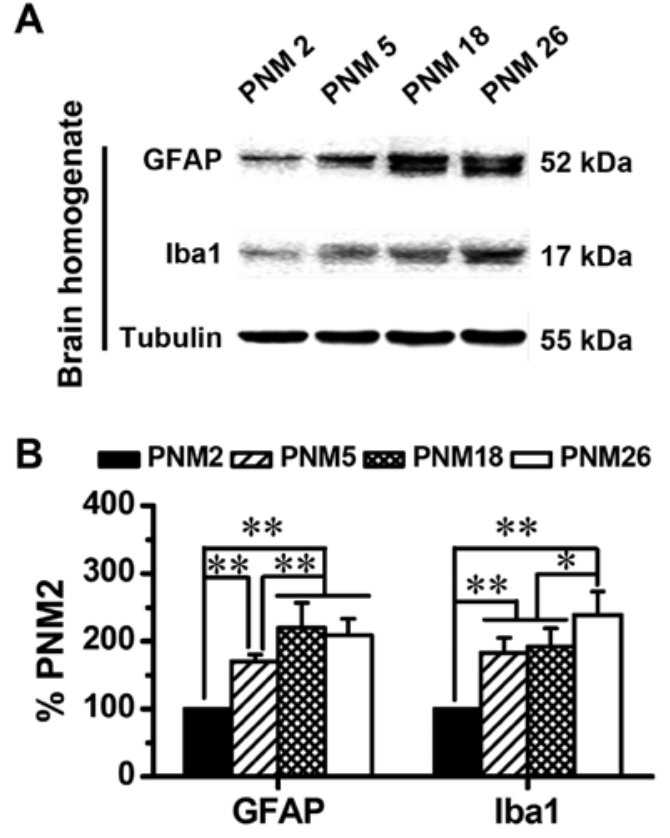

Figure 7. The upregulation of glial protein in the aging brain. (A) Expression of glial fibrillary acidic protein (GFAP) and Ibal based on western blot analysis in postnatal month 2 (PNM2), 5, 18 and 26 rat brain tissue homogenate ( $\mathrm{n}=4$ per time point). (B) Statistical analysis of western blot results. ${ }^{*} \mathrm{P}<0.05$; ${ }^{* *} \mathrm{P}<0.01$; Error bars: \pm SEM

rat, the non-cell-shaped staining was greater in PNM2 white matter (Fig. 5E-H, K and L). This dark background of white matter in PNM2 is probably caused by the staining of a neuropil that was formed by inactivated astrocytes. On the other hand, the activation of microglia was also significant in the grey and white matters (Fig. 6) and the upregulation of Ibal in aged grey matter was greater than that in white matter (Fig. 6N). Iba1-positive microglia were hardly detectable in the whole CNS of PNM2, which yielded the pale and achromatous appearance of slides. However, the number of Iba1-labeled microglia was markedly elevated in PNM18 brain and spinal cord. A 2- to 6-fold increase in the density of activated microglia was identified in the cerebral cortex, dentate gyrus of hippocampal formation, corpus callosum, internal capsule, spinal cord dorsal horn and spinal cord posterior funiculus, respectively (Fig. 6A-M).

Western blotting data showed an age-related accumulation of GFAP and Ibal in the brain (Fig. 7). The expression of GFAP and Ibal increased progressively with age, which doubled the levels of GFAP and Iba1 in PNM18 and PNM26 rat brains compared with those in PNM2 (Fig. 7B).

Correlation between age-related glial activation and myelin protein decline in aging. Considering that age-dependence was present both in the reduction of myelin proteins and the increase of glial markers, we evaluated whether these two alterations were correlated with each other. The main findings of the correlational analyses were that: i) the downregulation of Mbp showed a significant negative correlation with the expression of GFAP (Fig. 8A) and Iba1 (Fig. 8B) in the brain; ii) the level of expression of Mog in the brain was negatively correlated with the concentration of GFAP (Fig. 8C) and Iba1
(Fig. 8D), respectively. Stepwise multiple regression analysis, with the expression levels of GFAP and Iba1 as independent variables and the expression levels of Mog and Mbp as dependent variables, identified the concentration of GFAP and Ibal in the brain as independent factors for predicting the expression level of Mog (Adj. R-square $=0.861, \mathrm{P}<0.001$ ) and $\mathrm{Mbp}$ (Adj. R-square $=0.848, \mathrm{P}<0.001$ ). These data indicated that the upregulation of GFAP and Iba1, in other words, the activation of astrocytes and microglia, may contribute to the decline of myelin proteins in the aging process.

\section{Discussion}

Although there is evidence to show the effects of normal aging on the myelin sheath in human and animals, investigations have focused on the limited age-related changes in the expression of myelin proteins in CNS. Different experimental methods, different animals and different regions of interest in CNS could induce confusing and even conflicting results (12-14). Therefore, a comprehensive detailed investigation of the expression of myelin proteins in aged CNS is still needed. In the current study, a universal reduction in the expression of Mog and Mbp was identified in the CNS of aged rats through well-controlled mapping. This downregulation of myelin proteins was consistent with $\mathrm{Mbp}, \mathrm{P} 0$ decline in peripheral nerves $(15,16)$ and was likely to be a reason accounting for the reduced hematoxylin staining of aged myelin, which was identified in early studies on humans (3).

Mbp is one of the major abundant proteins in the myelin sheath, comprising $>30 \%$ of the total proteins in most species (17). Mbp is an extrinsic protein localized exclusively at the cytoplasmic surface in the major dense line of myelin sheath and is believed to be the principal protein stabilizing the major dense line of CNS myelin (17). Splits of the lamellae at the major dense line were found to be the most common morphological alterations in aged myelin sheath after a series of ultrastructural investigations $(11,18)$ and could probably be due to the age-related Mbp decrease observed in our study. On the other hand, Mog is a CNS myelin protein of great neuroimmunological interest, but its function remains to be determined (19). As yet, no other investigations have shown this age-related decrease of Mog. However, what changes were induced by this downregulation of Mog in aged CNS remain a mystery and should be further examined.

DAPI labeling of the nuclei in the corpus callosum indicated a significant increase of the glial cell number in aging brains. Considering that oligodendrocytes were the major cell type in corpus callosum, we assumed that elevation in the number of DAPI-labeled nuclei was mainly attributed to the increase of oligodendrocytes. This is consistent with the ultrastructural studies that were administered in the cerebral and visual cortexes of aging primates in the studies by Peters and Sethares and by Peters et al $(20,21)$. The aggregation of glial nuclei in the aged corpus callosum, observed in our study (Fig. 3), and the similar pair- or row-together of oligodendrocytes, which was found in aged monkey cortexes using electron microscopy $(20,22)$, suggested that oligodendrocytes proliferate with age and this proliferation leads to an increase in their number. However, DAPI labeling also indicated a decrease in the number of glial cells in aged 
A

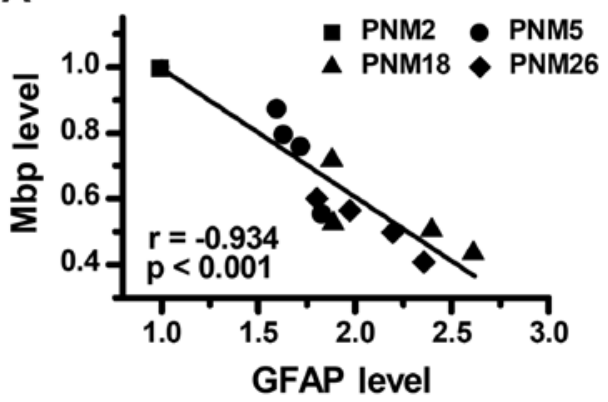

$c$

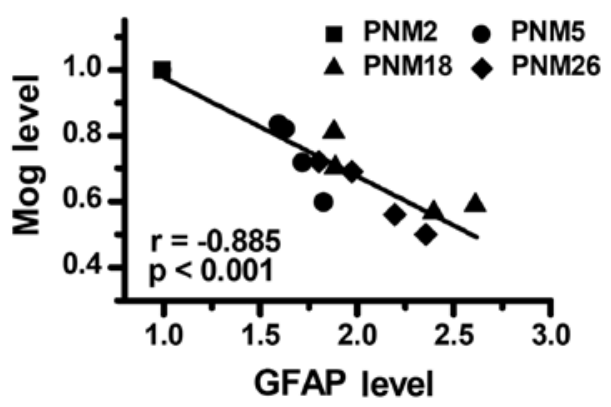

B

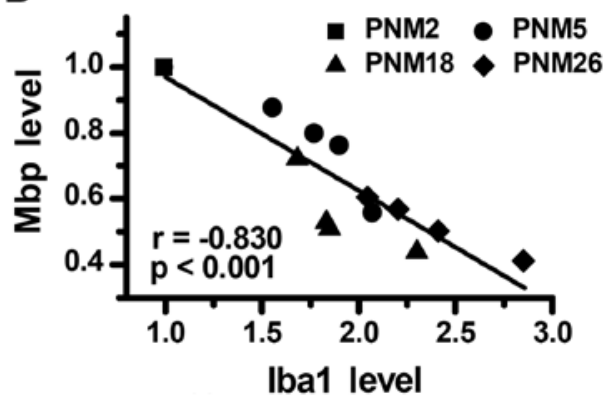

D

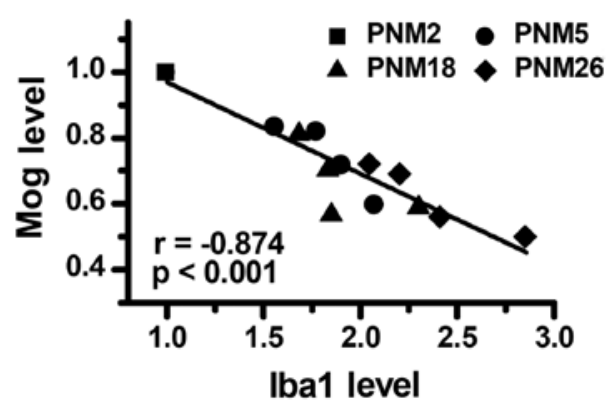

Figure 8. The negative correlation between the myelin protein breakdown and glia cell activation. (A) and (B) Expression of myelin basic protein (Mbp) and ionized calcium-binding adaptor molecule 1 (Iba1), respectively, in the brain were negatively correlated with the expression of GFAP. (C and D) Expression of myelin oligodendrocyte glycoprotein (Mog) in brain exhibits an inverse correlation to the expression of glial fibrillary acidic protein (GFAP) and Iba1.

spinal cord posterior funiculus. Further studies are needed to determine whether the increase in the number of oligodendrocytes in aging is universal in CNS.

Results of the immunohistochemical mapping and western blotting indicated a significant upregulation of GFAP and Iba1 in aged rat CNS. This increase of age-related GAFP (mRNA and protein expression levels) and hypertrophy of astrocytes in aged rodent brains were also identified in other studies $(23,24)$. However, previous reports have suggested that the number of astrocytes did not appear to increase or increased slightly in number during normal aging $(11,25)$, which was not consistent with the significant age-related increase in the number of astrocytes in the present study. The dark neuropil staining in the white matter, which was observed in PNM2 in our study (Fig. 5E-H, K and L) suggested that the inactive astrocytes expressed low levels of GFAP that were not easily detected by GFAP immunohistochemistry. Therefore, we assumed that the great changes of GFAP in our study were a good marker of astrocyte activation, but could not be used to evaluate the proliferation of astrocytes in aging.

On the other hand, Ibal was widely used as a microglial activation marker, since it is greatly upregulated in activated microglia (26). Based on Iba1 immunohistochemical staining, age-related activation of microglia was identified in our study, which was also detected in the brain of aged rats (27) and primates (28) using other microglial activation markers.

Our correlation analysis revealed that the age-related decrease of Mbp and Mog was highly correlated with the activation of astrocytes and microglia in aged rat CNS. As previous attention was mostly focused on the interaction between astrocytes/microglia and neurons, this negative correlation is relatively novel. A decreased neuroprotective capacity of aged astrocyte was also found by a previous in vitro study (29). This compromised neuroprotection of astrocytes, during aging, was believed to be associated with the reduced nerve growth factors in aging, such as FGF-2 and BDNF, which are released by astrocytes (30). Considering that sufficient nerve growth factors are also needed in maintaining the integrity of the myelin sheath (31), we assumed that the reduced nerve growth factors in aging astrocytes may contribute to the decline of myelin proteins. On the other hand, chronic activated microglia and astrocytes can induce damage by releasing highly toxic products, such as reactive oxygen intermediates, inflammatory cytokines and complementary factors (32). There is evidence showing that myelin proteolysis, in aging, is linked to calpain-1 and the complement system expressed in microglia (33). However, the exact mechanism of the decrease of age-related myelin proteins and the contribution of astrocytes and microglial activation in this decrease remain unknown. Further studies must be performed to explore the links between the downregulation of myelin proteins and activation of astrocytes and microglia.

In summary, a significant decline of myelin proteins in the whole CNS of aged rats was identified by immunohistochemical staining and western blotting. The decrease in myelin proteins was highly correlated with the age-related activation of astrocytes and microglia. Mog and Mbp mapping could be used as a good model to investigate the aging effects on myelin sheath in CNS. The correlation of myelin breakdown and glial activation in aging is able to provide new evidence concerning the connection of inflammation and myelin breakdown mechanism in age-related neurodegenerative diseases. 


\section{Acknowledgements}

This study was supported by grants from the Major State Basic Research Development Program of China (973 Program) (no. 2011CB504100, 2013BAI04B04) and the National Natural Science Foundation of China (no. 81171049).

\section{References}

1. Waxman SG: Conduction in myelinated, unmyelinated, and demyelinated fibers. Arch Neurol 34: 585-589, 1977.

2. Sherin JE and Bartzokis G: Human brain myelination trajectories across the life span: implications for CNS function and dysfunction. In: Handbook of the Biology of Aging. Masoro EJ and Austa SN (eds). 7th edition. Academic Press, San Diego, CA, pp333-346, 2011.

3. Lintl P and Braak H: Loss of intracortical myelinated fibers: a distinctive age-related alteration in the human striate area. Acta Neuropathol 61: 178-182, 1983.

4. Madden DJ, Bennett IJ, Burzynska A, Potter GG, Chen NK and Song AW: Diffusion tensor imaging of cerebral white matter integrity in cognitive aging. Biochim Biophys Acta 1822 386-400, 2012.

5. Bhadelia RA, Price LL, Tedesco KL, et al: Diffusion tensor imaging, white matter lesions, the corpus callosum, and gait in the elderly. Stroke 40: 3816-3820, 2009.

6. Verdu E, Ceballos D, Vilches JJ and Navarro X: Influence of aging on peripheral nerve function and regeneration. J Peripher Nerv Syst 5: 191-208, 2000.

7. Peters A: The effects of normal aging on myelinated nerve fibers in monkey central nervous system. Front Neuroanat 3: 11, 2009.

8. Bartzokis G: Alzheimer's disease as homeostatic responses to age-related myelin breakdown. Neurobiol Aging 32: 1341-1371, 2011.

9. Bohnen NI and Albin RL: White matter lesions in Parkinson disease. Nat Rev Neurol 7: 229-236, 2011.

10. Blasko I, Stampfer-Kountchev M, Robatscher P, Veerhuis R, Eikelenboom P and Grubeck-Loebenstein B: How chronic inflammation can affect the brain and support the development of Alzheimer's disease in old age: the role of microglia and astrocytes. Aging Cell 3: 169-176, 2004.

11. Peters A: The effects of normal aging on nerve fibers and neuroglia in the central nervous system. In: Brain Aging: Models, Methods, and Mechanisms. Riddle DR (ed). Boca Raton, FL. CRC Press, Taylor \& Francis, pp97-125, 2007.

12. Sloane JA, Hinman JD, Lubonia M, Hollander $W$ and Abraham CR: Age-dependent myelin degeneration and proteolysis of oligodendrocyte proteins is associated with the activation of calpain-1 in the rhesus monkey. J Neurochem 84: 157-168, 2003.

13. Xing Y, Samuvel DJ, Stevens SM, Dubno JR, Schulte BA and Lang H: Age-related changes of myelin basic protein in mouse and human auditory nerve. PLoS One 7: e34500, 2012.

14. Ciftci G, Yarim GF, Yarim M, et al: The effects of aging on central nervous system steroid prophiles and myelin basic protein in rats. Aging Clin Exp Res 24: 117-124, 2011.

15. Melcangi RC, Magnaghi V, Cavarretta I, Martini L and Piva F: Age-induced decrease of glycoprotein Po and myelin basic protein gene expression in the rat sciatic nerve. Repair by steroid derivatives. Neuroscience 85: 569-578, 1998.
16. Melcangi RC, Magnaghi V and Martini L: Aging in peripheral nerves: regulation of myelin protein genes by steroid hormones. Prog Neurobiol 60: 291-308, 2000.

17. Campagnoni AT and Campagnoni CW: Myelin basic protein gene. In: Myelin Biology and Disorders. Lazzarini RA (ed). Vol. 1, Elsevier Academic Press, San Diego, CA, pp387-400, 2004.

18. Peters A and Sethares C: Aging and the myelinated fibers in prefrontal cortex and corpus callosum of the monkey. J Comp Neurol 442: 277-291, 2002.

19. Pham-Dinh D, Dautigny A and Linington C: Myelin oligodendrocyte glycoprotein gene. In: Myelin Biology and Disorders. Lazzarini RA (ed). Vol. 1, Elsevier Academic Press, San Diego, CA, pp469-489, 2004

20. Peters A and Sethares C: Oligodendrocytes, their progenitors and other neuroglial cells in the aging primate cerebral cortex. Cereb Cortex 14: 995-1007, 2004.

21. Peters A, Josephson K and Vincent SL: Effects of aging on the neuroglial cells and pericytes within area 17 of the rhesus monkey cerebral cortex. Anat Rec 229: 384-398, 1991.

22. Peters A: Age-related changes in oligodendrocytes in monkey cerebral cortex. J Comp Neurol 371: 153-163, 1996.

23. Goss JR, Finch CE and Morgan DG: Age-related changes in glial fibrillary acidic protein mRNA in the mouse brain. Neurobiol Aging 12: 165-170, 1991.

24. Berciano MT, Andres MA, Calle E and Lafarga M: Age-induced hypertrophy of astrocytes in rat supraoptic nucleus: a cytological, morphometric, and immunocytochemical study. Anat Rec 243: 129-144, 1995.

25. Long JM, Kalehua AN, Muth NJ, et al: Stereological analysis of astrocyte and microglia in aging mouse hippocampus. Neurobiol Aging 19: 497-503, 1998.

26. Ito D, Imai Y, Ohsawa K, Nakajima K, Fukuuchi Y and Kohsaka S: Microglia-specific localisation of a novel calcium binding protein, Iba1. Brain Res Mol Brain Res 57: 1-9, 1998

27. Ogura K, Ogawa $M$ and Yoshida M: Effects of ageing on microglia in the normal rat brain: immunohistochemical observations. Neuroreport 5: 1224-1226, 1994.

28. Sloane JA, Hollander W, Moss MB, Rosene DL and Abraham CR: Increased microglial activation and protein nitration in white matter of the aging monkey. Neurobiol Aging 20: 395-405, 1999.

29. Pertusa M, Garcia-Matas S, Rodriguez-Farre E, Sanfeliu C and Cristofol R: Astrocytes aged in vitro show a decreased neuroprotective capacity. J Neurochem 101: 794-805, 2007.

30. Blasco I, Humpel C and Grubeck-Loebenstein B: Glial cells: astrocytes and oligodendrocytes during normal brain aging. In: Handbook of the Neuroscience of Aging. Hof PR and Mobbs CV (eds). Elsevier Academic Press, San Diego, CA, pp47-52, 2009.

31. de Groot DM, Coenen AJ, Verhofstad A, van Herp F and Martens GJ: In vivo induction of glial cell proliferation and axonal outgrowth and myelination by brain-derived neurotrophic factor. Mol Endocrinol 20: 2987-2998, 2006.

32. Godbout JP and Johnson RW: Age and neuroinflammation: a lifetime of psychoneuroimmune consequences. Immunol Allergy Clin North Am 29: 321-337, 2009.

33. Duce JA, Hollander W, Jaffe R and Abraham CR: Activation of early components of complement targets myelin and oligodendrocytes in the aged rhesus monkey brain. Neurobiol Aging 27: 633-644, 2006. 